\title{
Synchronous metastatic seminoma and primary retroperitoneal ganglioneuroma: case report and literature review
}

\author{
Jorg Michels, PhD, MRCP(UK); Nicholas van der Westhuizen, MD, FRCPC; ${ }^{\dagger}$ Alison Ross, MD
}

"Vancouver Island Centre, British Columbia Cancer Agency, Victoria, BC; † Pathologist, Department of Laboratory Medicine and Pathology, Vancouver Island Health Authority, Victoria, BC; §Surgeon, Department of Surgery, Vancouver Island Health Authority, Victoria, BC

Cite as: Can Urol Assoc J 2011;5(6):E109-E112; http://dx.doi.org/10.5489/cuaj.10164

\section{Abstract}

Testicular cancer is the most common malignancy in young men with seminomas comprising almost half of all germ cell tumours. Benign ganglioneuromas are rare tumours derived from the sympathetic nervous system. They usually occur in aldolescents and young adults and are predominantly located in the mediastinum and retroperitoneum. We report a case of a patient with synchronous metastatic testicular seminoma with retroperitoneal lymph node involvement and a benign retroperitoneal ganglioneuroma (RGN) which caused diagnostic and management challenges. The patient had a complete response following combination chemotherapy for his seminoma and subsequently underwent complete resection of his ganglioneuroma.

\section{Introduction}

Testicular cancers are uncommon, but they represent the most common malignancy in young men. Germ cell tumours account for $90 \%$ of testicular neoplasms, and about $40 \%$ to $60 \%$ of these are due to seminoma. Metastatic seminoma tends to involve retroperitoneal lymph nodes initially and, even then, cure rates remain very high $-90 \%$ of patients are relapse-free at 5 years. ${ }^{1,2}$ Following inguinal orchidectomy, radiation therapy to the para-aortic and ipsilateral pelvic area (dog-leg radiation) is standard for clinical stage II A/B seminoma (lymph node size $\leq 2 \mathrm{~cm}$ or $2-5 \mathrm{~cm}$, respectively). Chemotherapy using either 3 cycles of BEP (bleomycin, etoposide, cisplatin) or 4 cycles of EP (etoposide, cisplatin) is an alternative for patients with stage II seminoma who are not willing to undergo radiotherapy or for patients with larger retroperitoneal lymph node disease (II B/C, latter lymph node size $>5 \mathrm{~cm}){ }^{1,2}$

Ganglioneuromas are rare benign tumours derived from sympathetic neural crest cells. This histologically fully differentiated tumour is distinct from its malignant counterpart, ganglioneuroblastoma, by the absence of immature elements, atypia or necrosis. Ganglioneuromas usually occur in aldolescents and young adults. They are commonly detected as incidental findings on imaging of the abdomen/retroperitoneum or mediastinum. Patients are often asymptomatic or develop symptoms related to local growth. Tumours can be hormonally active (catecholamines, vasoactive intestinal peptide) resulting in symptoms, such as hypertension, palpitations or diarrhea. Metastases are exceedingly rare and complete surgical resection is the treatment of choice in suitable patients. ${ }^{3,4}$

We report an unusual case of a patient with metastatic seminoma to retroperitonial lymph nodes and concurrent retroperitoneal ganglioneuroma which posed a diagnostic and therapeutic challenge. The patient had a complete clinical response following 4 cycles of EP for seminoma and subsequently underwent successful resection of the retroperitoneal ganglioneuroma. The literature is reviewed.

\section{Case report}

A 35-year-old man presented with a 2-month history of right testicular discomfort and swelling. Testicular ultrasound demonstrated a right testicular tumour. Baseline serum markers revealed normal levels of AFP (alpha fetoprotein) and LDH (lactate dehydrogenase) and a mildly elevated bHCG (beta human chorionic gonadotropin) of $3.7 \mathrm{IU} / \mathrm{L}$ (upper limit of normal: $2.0 \mathrm{IU} / \mathrm{L}$ )

Radical right orchidectomy revealed a $4.5-\mathrm{cm}$ pure classical seminoma (Fig. 1, parts A and B) of pathological stage pT1 without rete testis infiltration or lymphovasular space invasion. Staging computed tomography (CT) of chest/ abdomen and pelvis demonstrated a retropancreatic lesion $(4 \times 3.6 \times 5.9 \mathrm{~cm}$, transverse/anterior-posterior/craniocaudal dimensions, respectively) and a separate precaval lesion $(1.7 \times 1.5 \times 2.1 \mathrm{~cm})$ which suggested metastases (Fig. 2, part A). The patient received 4 cycles of standard dose etoposide $\left(500 \mathrm{mg} / \mathrm{m}^{2}\right)$ in combination with cisplatin $\left(100 \mathrm{mg} / \mathrm{m}^{2}\right)$, 
Michels et al.

given over 5 days and repeated at 3 weekly intervals without delay. His bHCG level normalized, and the post-treatment $\mathrm{CT} /$ magnetic resonance imaging (MRI) demonstrated complete resolution of the retroperitoneal precaval lymph node metastasis, but the retropancreatic lesion remained the same size (Fig. 2, part B). Low signal enhancement on MRI raised the possibility that this was a fibrous lesion.

The clinical suspicion of a seminoma-independent retroperitoneal process was raised and the patient underwent a CT-guided fine needle aspirate (FNA) followed by a linear endoscopic ultrasound scan (EUS) guided FNA; these tests revealed non-diagnostic and normal tissue only. The lesion exhibited clinically benign features. Surgical resection was considered difficult with intimate proximity to pancreas and blood vessels (superior mesenteric artery/vein). An open surgical biopsy was undertaken first. Intraoperative findings revealed a well-circumscribed and potentially resectable tumour. Pathology confirmed findings consistent with benign ganglioneuroma without malignant or seminomatous elements. Immunohistochemical staining for phosphorylated neurofilament (PNF) showed numerous bundles of PNF-positive processes and occasion positive neuronal cell bodies. The well circumscribed, solid mass in the retropancreatic location was subsequently completely excised. Pathology demonstrated a $7 \times 5 \times 4.2 \mathrm{~cm}$ thinly encapsulated tumour composed of a background of Schwann cells (resembling a neurofibroma) admixed with scattered mature ganglion cells diagnostic of a ganglioneuroma (Fig. 1, parts $\mathrm{C}$ and D). Interestingly, the patient complained of episodes of palpation and night sweats pre-dating the removal of his ganglioneuroma. Repeated urine collection for norepinephrine/epinephrine/dopamine levels were within normal limits, as was his serum testosterone level.

The patient recovered well from his surgery and is relapsefree at 2.5 years.

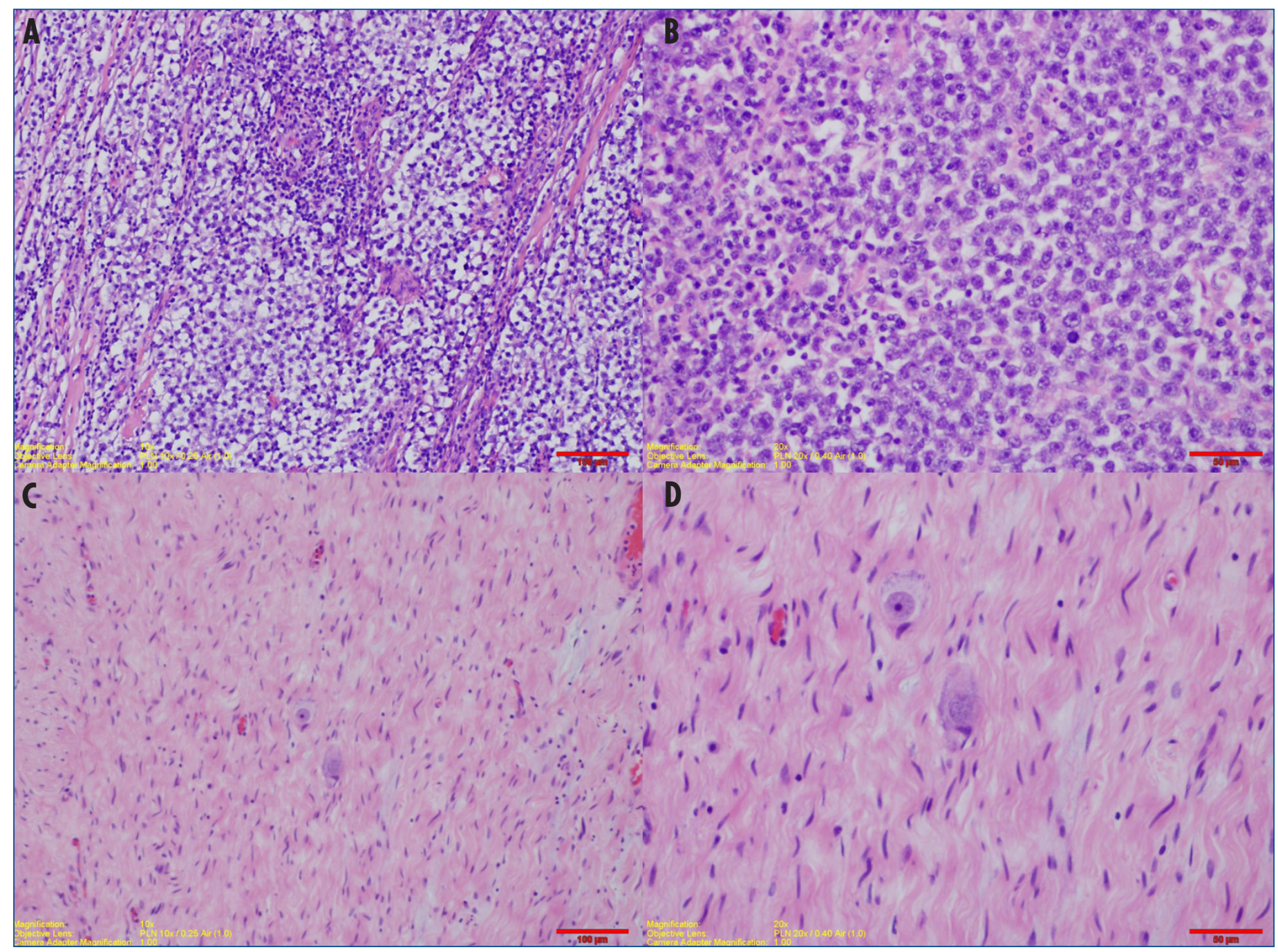

Fig. 1. Pathology: representative microscopic section of seminoma $(A / B)$ and ganglioneuroma $(C / D)$ are shown at low and high power field. 

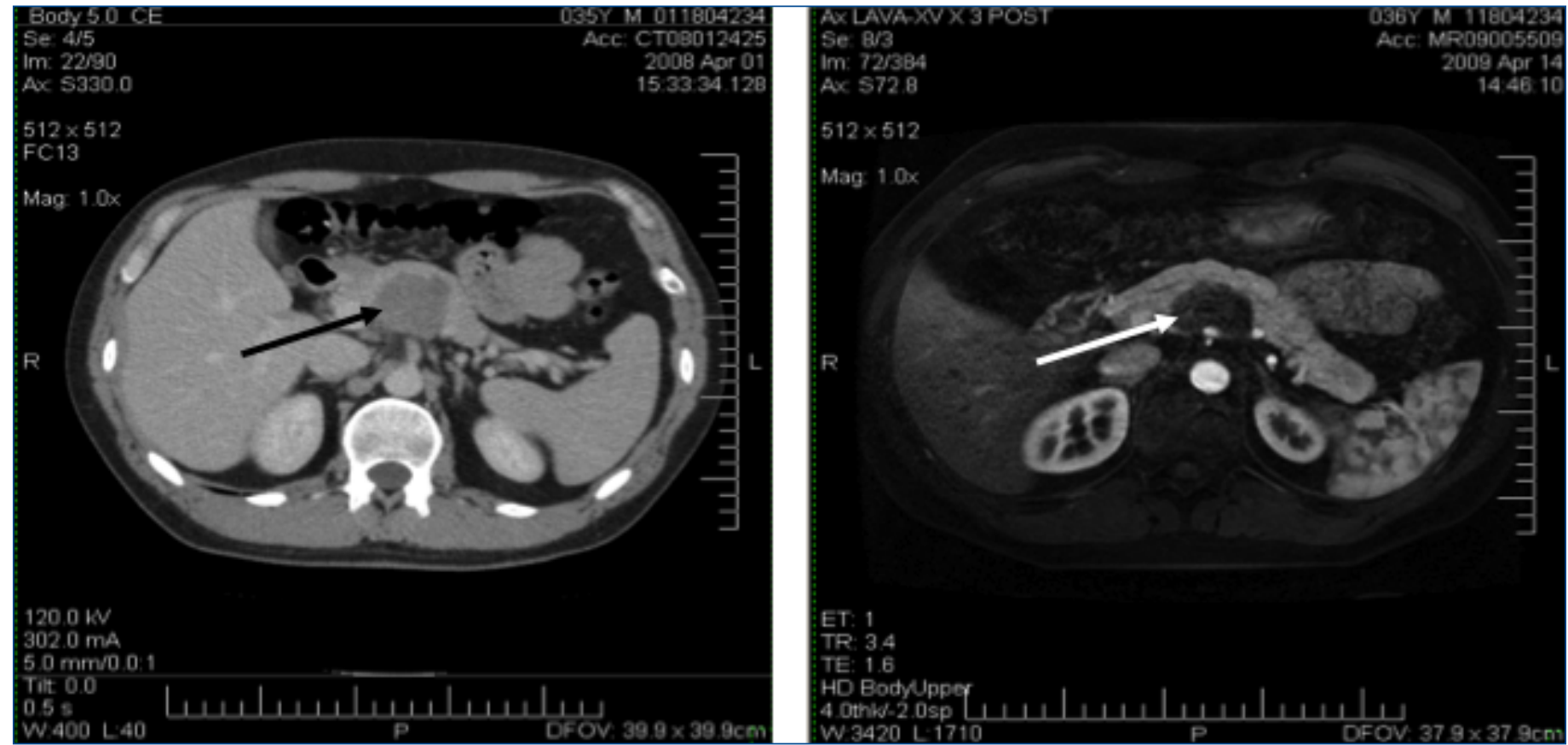

Fig. 2. Axial computer tomographic (CT)/magnetic resounance images (MRI). Corresponding imaging sections of the retroperitoneal lesion (marked with arrow) on CT before (A) and MRI after chemotherapy (B) are demonstrated. The low signal enhancement on MRI of the retroperitoneal ganglioneuroma is evident.

\section{Discussion}

The presented patient appears to be the first detailed case report of a synchronous metastatic seminoma and ganglioneuroma in the literature. One series on [18F]-2-fluoro-2deoxy-D-glusose positron emission tomography (18FDGPET) in 54 testicular cancer patients found 1 seminoma patient who had a ganglioneuroma on retroperitoneal lymph node dissection. ${ }^{5}$ This patient had a persistent large tumour despite chemotherapy, similar to our case.

The rarity of such a coincidental tumour diagnosis is not surprising taking into account that only small case series are available of RGNs in adults and the incidence rate of testis cancer is 9 per 100000 men per year in Canada.,3,6

Metastatic seminoma can spread to the retroperitoneal lymph node regions involving precaval, paracaval, interaortocaval, pre- and para-aortic stations. ${ }^{7}$ Staging imaging in our patient revealed a precaval enlarged lymph node and, perhaps in a somewhat unusual location, a retropancreatic soft tissue mass thought to be metastatic seminoma initially. Seminoma is an exquisitely chemosensitive malignancy, and postchemotherapy residual masses can be encountered with large initial tumour masses. Our patient developed a complete resolution of the enlarged precaval lymph node. He had an unchanged retropancreatic tumour mass which contended with the expected response pattern in seminoma.

Findings on a post-treatment CT and MRI were non-specific, but suggested a fibrous and clinically benign appearing retropancreatic process with possible infiltration into the pancreas. The differential diagnosis in this case included ganglioneuroma, ganglioneuroblastoma, schwannoma, neurofibroma, neuroblastoma and testis cancer. A definitive tissue diagnosis was required. Although repeated imaging guided FNA was non-contributory in our case, such an approach can be successful. ${ }^{8}$

Surgical resection was initially considered as extensive (including partial pancreatectomy) due to the close location of the lesion to the pancreas and major vasculature. In addition, complete surgical resection was deemed unlikely to be curative if a malignant tumour were to be found. The patient therefore underwent open biopsy first. This approach was very helpful as it established the diagnosis of a benign ganglioneuroma. It also indicated the resectability of the circumscribed, encapsulated and mobile mass. Radical excision may be unnecessary if undue surgical morbidity is anticipated due to the favourable overall outlook of ganglioneuromas. ${ }^{4,9}$ However, surgical resection is generally indicated to rule out immature neuroblastic elements and potential aggressive behavior and to avoid the potential local complications arising from a growing tumour mass and the catecholamine synthesis of RGN. ${ }^{3,10}$ Our patient developed symptoms suggestive for catecholamine secretion from RGN. His endocrine/metabolic profile was normal and the reported symptoms were likely related to his previous chemotherapy for seminoma. Ultimately, our patient underwent uneventful complete resection. 
Synchronous metastatic seminoma \& primary retroperitoneal ganglioneuroma

\section{Conclusion}

Metastatic seminoma with assumed metastasis in an atypical location can indicate an independent synchronous process. Treatment for seminoma in general takes precedence due to the very high cure rate. Synchronous benign RGNs are exceedingly rare in testis cancer patients and may be difficult to diagnose. Surgical resection is the treatment of choice, which should be considered in patients who are reasonable surgical candidates.

Competing interests: None declared.

This paper has been peer-reviewed.

\section{References}

1. Schmoll HJ, Souchon R, Krege S, et al. European consensus on diagnosis and treatment of germ cell cancer: a report of the European Germ Cell Cancer Consensus Group (EGCCCG). Ann Oncol 2004;15:1377-99.

2. NCCN, Testicular Cancer. NCCN (National Comprehensive Cancer NetWork) Clinical Practice Guidelines in Oncology, 2010. V.2.2010. www.nccn.org. Accessed November 16, 2011.

3. Geoerger B, Hero B, Harms D, et al. Metabolic activity and clinical features of primary ganglioneuromas. Cancer 2001;91:1905-13.

4. Papavramidis TS, Michalopoulos N, Georgia K, et al. Retroperitoneal ganglioneuroma in an adult patient: a case report and literature review of the last decade. South Med J 2009;102:1065-7.

5. Muller-Mattheis $V$, Reinhardt M, Gerharz CD, et al. [Positron emission tomography with [18 F]-2-fluoro-2deoxy-D-glucose (18FDG-PET) in diagnosis of retroperitoneal lymph node metastases of testicular tumours]. Der Urologe. Ausg 1998;37:609-20.

6. Public Health Agency of Canada. Cancer in Young Adults in Canada. http://www.phac-aspc.gc.ca/ publicat/cyac-cjac06/pdf/cyac-cjac-2006_e.pdf, p38-39. Accessed December 2, 2011.

7. DeVita VH, Rosenberg SA. Cancer, Principles \& Practice of Oncology. 7th ed., chapter 31; 2005:1269ff.

8. Layfield U, Glasgow BJ, Du Puis MH, et al. Aspiration cytology and immunohistochemistry of a pheochromocytoma-ganglioneuroma of the adrenal gland. Acta Cytol 1987;31:33-9.

9. Nakanishi S, Segawa T, Aragaki R, et al. [Retroperitoneal ganglioneuroma presenting as a soft tissue mass surrounding the aorta: report of a case]. Hinyokika Kiyo 2005;51:253-5.

10. Cronin EM, Coffey JC, Herlihy D, et al. Massive retroperitoneal ganglioneuroma presenting with small bowel obstruction 18 years following initial diagnosis. Ir J Med Sci 2005;174:63-6.

Correspondence: Dr. Jorg Michels, BC Cancer Agency, Vancouver Island Centre, 1410 Lee Avenue, Victoria, BC V8R 6V5; fax: 250-519-2017; imichels@bccancer.bc.ca 